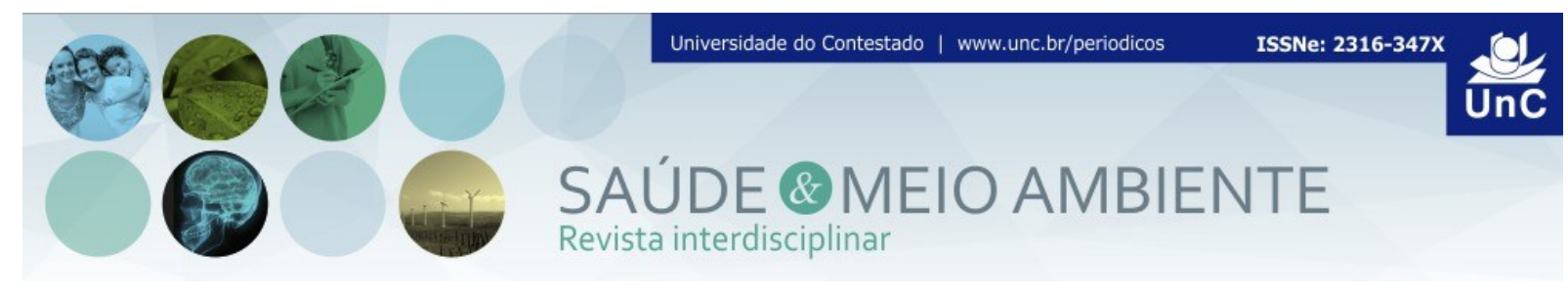

\title{
AVALIAÇÃO DA EXPOSIÇÃO AO MONÓXIDO DE CARBONO EM TRABALHADORES DE INDÚSTRIAS DE CERÂMICAS NO BAIRRO JADERLÂNDIA EM SANTARÉM-PARÁ
}

\author{
Karen Cristini Yumi Ogawa Maestri ${ }^{1}$ \\ Elizângela Silva Da Costa ${ }^{2}$ \\ Brenda Mayara Diniz Guimarães ${ }^{3}$ \\ Carla Daiane Auzier ${ }^{4}$ \\ Cassiano Junior Saatkamp 5 \\ Arimar Chagas de Almeida ${ }^{6}$ \\ Régis Piloni Maestri
}

\begin{abstract}
RESUMO: A indústria ceramista é um dos âmbitos mais agravantes da poluição ambiental, devido ao alto índice de fumaça contendo substâncias químicas, como o monóxido de carbono (CO) que é um dos poluentes mais comuns, resultantes da atividade desse setor. O que leva a expor seus trabalhadores de diferentes ocupações a esse agente tóxico. O objetivo do presente estudo foi avaliar a concentração de carboxihemoglobina $(\mathrm{COHb})$ em relação à exposição ao $\mathrm{CO}$ em trabalhadores de indústria de cerâmicas no bairro Jaderlândia em Santarém - Pará. A amostra foi constituída por 60 trabalhadores. A partir das análises, verificou-se que $17 \%$ (10/60), apresentaram níveis de $\mathrm{COHb}$ acima do que preconiza a Norma Regulamentadora 7 (NR -7) do Ministério do Trabalho. Cabe ressaltar, que o limite aceitável é de no máximo 3,5\%. A mensuração dos níveis de $\mathrm{COHb}$ nos trabalhadores mostrou que $83 \%(50 / 60)$ apresentou níveis dentro dos limites aceitáveis, de acordo com o que preconiza a NR-7. Desta forma, é importante manter um sistema periódico de monitoramento, fazendo-se necessárias a elaboração e a implementação de um programa de acompanhamento e controle sistemático dos poluentes do ar e seus efeitos sobre a saúde humana.
\end{abstract}

Palavras-chave: Intoxicação ao monóxido de carbono. Carboxihemoglobina. Exposição Ocupacional.

\footnotetext{
${ }^{1}$ Mestrado em Doenças Tropicais pelo Núcleo de Medicina Tropical, Brasil (2012). Trabalha no Biomedical and Biopharmaceutical Research. E-mail: karenmaestri@hotmail.com.br

${ }^{2}$ Graduanda em Farmácia. Instituto Esperança de Ensino Superior, IESPES, Brasil. E-mail: elizangela.costa.7@hotmail.com

3 E-mail: brendha guimaraes@hotmail.com

${ }^{4}$ Graduação em Farmácia pelo Instituto Esperança de Ensino Superior, Brasil (2014). E-mail: mille.patty@hotmail.com

${ }^{5}$ Mestrado em Bioengenharia pela Universidade Camilo Castelo Branco, Brasil (2014). Farmacêutico Bioquímico do LABORATÓRIO SANTOS , Brasil. E-mail: juniorsc7@yahoo.com.br

${ }^{6}$ MBA em Administração Hospitalar e Serviços de Saúde pela Faculdade INESP, Brasil (2010). Coordenador de Farmácia do Hospital Regional do Baixo Amazonas Dr. Waldemar Pena, Brasil. Email: arimaralmeidarc@yahoo.com.br

${ }^{7}$ Mestrado em Doenças Tropicais pela Universidade Federal do Pará, Brasil (2012). Trabalha no Instituto Esperança de Ensino Superior, Brasil. E-mail: regismaestri@hotmail.com
} 


\title{
EVALUATION OF EXPOSURE CARBON MONOXIDE IN CERAMIC INDUSTRIES WORKERS IN NEIGHBORHOOD IN JADERLÂNDIA SANTARÉM-PARA
}

\begin{abstract}
The ceramics industry is one of the most aggravating aspects of environmental pollution due to high levels of smoke containing chemicals such as carbon monoxide (CO) which is one of the most common pollutants resulting from the activity of this sector. Which leads to expose their workers of different occupations to this toxic agent. The aim of this study was to evaluate the concentration of carboxyhemoglobin $(\mathrm{COHb})$ relative to $\mathrm{CO}$ exposure in the ceramics industry workers in Jaderlândia district of Santarém - Pará. The sample consisted of 60 workers. From the analysis, it was found that $17 \%(10 / 60)$ had $\mathrm{COHb}$ levels above what advocates Regulatory Standard 7 (NR -7) of the Ministry of Labour. It is noteworthy that the acceptable limit is a maximum of $3.5 \%$. The measurement of $\mathrm{COHb}$ levels in workers showed that $83 \%(50 / 60)$ showed levels within acceptable limits, according to which calls NR 7 . Thus, it is important to maintain a regular monitoring system, making it necessary the development and implementation of a monitoring program and systematic control of air pollutants and their effects on human health.
\end{abstract}

Key words: Carbon monoxide poisoning. Carboxyhemoglobin. Occupational exposure.

\section{INTRODUÇÃO}

Durante o processo respiratório, cerca de $97 \%$ das moléculas de $\mathrm{O}_{2}$ levadas do pulmão para os tecidos se combinam quimicamente com a $\mathrm{Hb}$, por ação imediata, os 3\% restantes são transportados em estado dissolvido no plasma e nas células (GUYTON, 2008). O ferro (Fe) é conduzido para a molécula de Hb através da sua proteína transportadora a siderofilina ou transferrina, onde se liga ao grupo heme da molécula. Portanto, $\mathrm{o} \mathrm{O}_{2}$ une-se a $\mathrm{Hb}$ devido ao $\mathrm{Fe}$ presente na parte não protéica da molécula, formando assim a oxihemoglobina (HbO) (LORENZI, 2011). Deste modo, a corrente sanguínea leva a $\mathrm{HbO}$ até as células dos tecidos de todo o corpo e ao passar pelas células do corpo o $\mathrm{O}_{2}$ vai sendo separado para fazer parte das reações químicas no meio celular. Dessa forma, a $\mathrm{Hb}$ fica novamente livre e o processo é reiniciado (SALLES, 2013).

O monóxido de carbono ( $\mathrm{CO}$ ) é considerado toxicologicamente um asfixiante químico e insalubre proveniente da combustão incompleta de materiais que contenham carbono. Suas principais fontes de liberação são as usinas termoelétricas a carvão, veículos automotores, queima de materiais orgânicos, queima de tabaco entre outros (BOHM, 2014; CASTRO, GOUVEIA e CEJUDO, 2003).

O CO também reage com a molécula de $\mathrm{Hb}$ por um processo muito parecido ao do $\mathrm{O}_{2}$, formando desta forma, a carboxihemoglobina ( $\left.\mathrm{COHb}\right)$. Quando em 
elevadas concentrações no ar inspirado, o CO entrará em uma competição no sangue, com $\mathrm{O}_{2}$ pelas moléculas de $\mathrm{Hb}$. A consequência dessa ação é que o $\mathrm{Fe}$ da $\mathrm{Hb}$ liga-se com mais força ao $\mathrm{CO}$ do que ao $\mathrm{O}_{2}$, este processo ocorre através de uma ligação covalente estável. Desta forma, a $\mathrm{Hb}$ dificilmente estará livre para transportar $\mathrm{O}_{2}$. Portanto, quanto maior a quantidade de $\mathrm{CO}$ inalado, maior será a quantidade de moléculas de $\mathrm{Hb}$ impossibilitadas de transportar $\mathrm{O}_{2}$, tendo como consequências graves danos à saúde (MOREAU e SIQUEIRA, 2011).

O CO também se apresenta no corpo em resposta de processos fisiológicos das células. Entretanto, através de estudos científicos tem sido revelado nos últimos anos, que o CO é um sinalizador celular que desempenha diversas ações, desencadeadas a partir de sua produção endógena, através da enzima heme oxigenase. Seu papel fisiológico se dá no sistema cardiovascular, sistema nervoso, respiratório, reprodutor e gastrointestinal (PINA e FURLAN, 2007).

Para o bom funcionamento dos órgãos, é necessário a oxigenação das células. Entretanto, alguns órgãos precisam de mais oxigenação que outros. Assim, o sistema nervoso central é o maior consumidor de $\mathrm{O}_{2}$, tornando-o muito sensível a falta deste gás (BOHM, 2014).

A formação da $\mathrm{COHb}$ e a manifestação de sinais e sintomas clínicos dependem principalmente da concentração do $\mathrm{CO}$ no ar atmosférico inspirado, do período de exposição, do tipo de atividade física e da susceptibilidade individual (GILMAN, 2003).

No setor de construção civil estão inseridas indústrias de cerâmicas as quais fazem parte de um cenário agravante que contribui para a poluição ambiental, influenciando inclusive a contaminação natural, onde o $\mathrm{CO}$ é o poluente mais comum resultante da atividade desse setor. Algumas dessas fábricas devido ao crescimento desordenado do ramo apresentam uma precariedade nas condições de trabalho e pouco conhecimento sobre estrutura de segurança do trabalho (GOMES, 2012).

Operários que trabalham nas atividades cerâmicas decorrentes da falta de proteção podem estar sujeitos a algumas doenças relacionadas à intoxicação crônica ao CO, como cefaleia, náuseas ou vômitos, insônia, fadiga, tonturas, entre outros. E sintomas incluídos na intoxicação aguda causada por exposição curta e rápida, bem como, distúrbios respiratórios, diminuição dos reflexos, entre outros. O grau de exposição ao $\mathrm{CO}$ é avaliado por meio da $\mathrm{COHb}$ que é considerada um indicador biológico (IB) quando exposta a este toxicante (OGA, CAMARGO e BATISTUZZO, 2008).

A concentração natural de $\mathrm{COHb}$ no sangue de pessoas não fumantes é de aproximadamente $0,5 \%$ (valor de referência para essa população), devido a produção endógena de CO durante a primeira etapa da degradação do grupo heme. A estimativa percentual da $\mathrm{COHb}$ no sangue da população é empregado como IB de exposição ao $\mathrm{CO}$ na monitoração biológica (MB) (OGA, CARMARGO e BATISTUZZO, 2003). 
Desta forma o presente estudo tem como objetivo avaliar a concentração de $\mathrm{COHb}$ em relação a exposição ao $\mathrm{CO}$ em trabalhadores de indústrias de cerâmicas no bairro Jaderlândia em Santarém - Pará.

\section{MÉTODOS}

Foi realizada uma pesquisa de campo e biomédica, de abordagem quantitativa, com características descritivas, com uma amostra de 60 participantes de ambos os sexos. Os participantes estudados foram constituídos por trabalhadores de cinco cerâmicas localizadas no bairro Jaderlândia em Santarém Pará.

\section{Determinação da carboxihemoglobina}

Para a determinação da $\mathrm{COHb}$ em sangue, foi utilizada a técnica descrita por Beutler e West(1984). O princípio do método consiste na redução da $\mathrm{Hb}$ com ditionito de sódio formando a hemoglobina reduzida $\left(\mathrm{Hb}_{\mathrm{red}}\right)$, a desoxihemoglobina.

\section{Fatores de calibração para determinação de carboxihemoglobina por espectrofotometria UV/Visível}

Para obtermos o fator de calibração foi utilizado sangue de uma pessoa não fumante e não exposta ocupacionalmente ao $\mathrm{CO}$, sendo realizada antes da coleta uma entrevista para obter a amostra desejada.

Em tubo de ensaio contendo $12 \mathrm{~mL}$ de solução hemolisante, adicionou-se $100 \mu \mathrm{L}$ de sangue de não fumante e agitou por inversão 5 vezes, obtendo a solução hemolisada. Em seguida em 2 balões volumétricos ( $A$ e B) de $25 \mathrm{~mL}$ cada, adicionouse $2 \mathrm{~mL}$ da solução hemolisada. Em seguida, no balão $A$ borbulhou $\mathrm{CO}$, gerado em laboratório, por 20 minutos excluindo os primeiros 5 minutos, e no $\mathrm{B}$ borbulhou $\mathrm{O}_{2}$ proveniente de cilindro de gás por 15 minutos, completou-se o volume de cada balão volumétrico com solução redutora, tampou e homogeneizou por inversão 5 vezes. Após, fez-se a leitura nas Abs 420 e 432 nm, zerando o aparelho com solução redutora em cada comprimento de onda, obtendo os fatores de calibração do aparelho $F_{1}, F_{2}$ e $F_{3}$. Esses fatores foram mensurados a partir das razões das Abs médias obtidas. Calculados conforme as equações 1,2 , e 3 , respectivamente. Os valores dos fatores $F$ obtidos na pesquisa foram: $F 1=1, F 2=0,6068, F 3=1,3250$. 
$\begin{array}{ll}\text { 1) } \mathrm{F} 1=\frac{\text { Abs Hbred } 432}{\text { Abs Hbred } 420} & \text { 2) } \mathrm{F} 2=\frac{A b s \mathrm{COH} b 432}{\text { Abs Hbred } 420}\end{array}$

3) $\mathrm{F} 3=\frac{\text { Abs COHb } 420}{\text { Abs Hbred } 420}$

$\mathrm{Hb}$ red - Hemoglobina reduzida

Abs - Absorbância

Equação 1: Determinação do fator de calibração $\mathrm{f}_{1}$, onde abs $h b$ red 432/abs hbred 420,são as absorbâncias da solução hemolisada ventilada com $\mathrm{O}_{2}$ e obtidas através das leituras 432 e $420 \mathrm{~nm}$.

Equação 2: Determinação do fator de calibração $f_{2}$, onde abs cohb 432/abs hb red 420, são as absorbâncias da solução hemolisada ventilada com coe obtidas através das leituras 432 e $420 \mathrm{~nm}$.

Equação 3: Determinação do fator de calibração $f_{3}$, onde abs cohb 420 / abs $h b$ red 420 , são as absorbâncias da solução hemolisada, obtidas através da leitura em $420 \mathrm{~nm}$. sendo o abs cohb a solução hemolisada ventilada com coe abs $h b$ red a solução hemolisada ventilada com $\mathrm{O}_{2}$.

\section{PROCEDIMENTO DA DETERMINAÇÃO DA PORCENTAGEM DE CARBOXIHEMOGLOBINA POR ESPECTROFOTOMETRIA UVIVISÍVEL}

Para maior precisão dos resultados foi utilizado como padrão o sangue de uma pessoa não fumante e não exposta ocupacionalmente ao $\mathrm{CO}$, sendo realizada antes da coleta uma entrevista, em seguida feito a análise da amostra tendo como critério para o resultado, apresentar nível de $\mathrm{COHb}$ de $1 \%$ em sangue, que segundo a NR 7 esse valor é definido como possível de ser encontrado em populações não expostas ocupacionalmente e não fumantes.

Após preparo das soluções: hemolisante (solução tampão / água destilada) e redutora ( $87,5 \mathrm{mg}$ de ditionito de sódio em $70 \mathrm{~mL}$ de solução tampão), adicionou-se $100 \mu \mathrm{l}$ de sangue em tubo de ensaio contendo $12 \mathrm{~mL}$ de solução hemolisante. A seguir, os tubos foram tampados e homogeneizados por inversão 5 vezes, deixando em repouso por 5 minutos em temperatura ambiente. Em seguida, $200 \mu \mathrm{L}$ desta solução foi pipetada para o tubo de ensaio de $5 \mathrm{ml}$ contendo $2,3 \mathrm{ml}$ da solução redutora. Homogeneizou-se novamente por inversão 5 vezes. Após novo período de repouso de 5 minutos, fez-se a leitura em espectrofotômetro UV/Visível nos 
comprimentos de onda 420 e $432 \mathrm{~nm}$, zerando o aparelho com solução redutora em cada comprimento de onda. A partir da média das Abs de cada amostra a porcentagem de $\mathrm{COHb}$ foi calculada conforme a equação 4.

$$
\begin{gathered}
\% \mathrm{COHb}=\frac{1-(\mathrm{AR} \times \mathrm{F} 1)}{\mathrm{AR}(\mathrm{F} 2-\mathrm{F} 1)-\mathrm{F} 3+1} \times 100 \\
\mathrm{AR}=\frac{\mathrm{Abs} 420}{\mathrm{Abs} 432} \text { e F1, F2 e F3 são os fatores de calibração do aparelho. }
\end{gathered}
$$

Equação 4: Determinação dos níveis de carboxihemoglobina

As amostras foram analisadas em duplicata, onde foram aceitas quando os valores de cada amostra alcançaram diferença menor ou igual a 10\%. Quando contrário, o procedimento é repetido após serem investigadas as prováveis fontes de erro.

\section{RESULTADOS E DISCUSSÃO}

\section{PERFIL OCUPACIONAL DOS TRABALHADORES CERAMISTAS}

No período de fevereiro a março de 2014, foram realizadas 60 entrevistas para traçar o perfil dos trabalhadores quanto ao sexo, faixa etária, tempo de trabalho, turno de trabalho, jornada de trabalho, setor de trabalho e utilização de equipamento de proteção individual (EPI) (máscara), conforme a tabela 1.

Tabela 1 - Perfil ocupacional das variáveis dos trabalhadores relacionada à porcentagem de

\begin{tabular}{|c|c|c|c|c|c|}
\hline \multirow[t]{2}{*}{ VARIÁVEÍS } & & \multicolumn{2}{|c|}{$\begin{array}{l}<3,5 \text { de } \mathrm{COHb} \\
\mathrm{n}=50\end{array}$} & \multicolumn{2}{|c|}{$\frac{>3,5 \text { de } \mathrm{COHb}}{\mathrm{n}=10}$} \\
\hline & & & $\%$ & $\mathrm{~N}^{0}$ & $\%$ \\
\hline \multirow[t]{2}{*}{ SEXO } & Masculino & 41 & 82 & 10 & 100 \\
\hline & Feminino & 9 & 18 & 0 & 0 \\
\hline \multirow[t]{3}{*}{ FAIXA ETÁRIA } & 18 a 25 anos & 24 & 48 & 3 & 30 \\
\hline & 26 a 31 anos & 11 & 22 & 3 & 30 \\
\hline & 32 a 61 anos & 15 & 30 & 4 & 40 \\
\hline TEMPO DE TRABALHO & $<1$ ano & 21 & & 2 & 20 \\
\hline
\end{tabular}
carboxihemoglobina

Saúde Meio Ambient. v. 4, n. 2, p. 90-102, jul./dez. 2015 


$\begin{array}{lllll} & & 42 & & \\ 1 \text { a } 2 \text { anos } & 6 & 12 & 2 & 20 \\ 3 \text { a } 4 \text { anos } & 6 & 12 & 3 & 30 \\ >5 \text { anos } & 17 & 34 & 3 & 30\end{array}$

TURNO DE TRABALHO

$\begin{array}{lllll}\text { Manhã e Tarde } & 47 & 94 & 9 & 90 \\ \text { Manhã } & 1 & 2 & 0 & 0 \\ \text { Tarde } & 1 & 2 & 0 & 0 \\ \text { Noite } & 1 & 2 & 1 & 10\end{array}$

JORNADA DE TRABALHO

$\begin{array}{lllll}4 \text { horas } & 1 & 2 & 0 & 0 \\ 8 \text { horas } & 44 & 88 & 9 & 90 \\ 12 \text { horas } & 2 & 4 & 1 & 10 \\ \text { Mais de 12 horas } & 3 & 6 & 0 & 0\end{array}$

SETOR DE TRABALHO

$\begin{array}{lllll}\text { Serviços Gerais } & 36 & 72 & 6 & 60 \\ \text { Forno } & 9 & 18 & 4 & 40 \\ \text { Administrativo } & 5 & 10 & 0 & 0\end{array}$

EPI (MÁSCARA)

\begin{tabular}{lllll} 
Sim & 28 & 56 & 7 & 70 \\
Não & 22 & 44 & 3 & 30 \\
\hline
\end{tabular}

Em um estudo realizado por Sousa (2009), no ambiente das indústrias de cerâmicas há o predomínio do gênero masculino, o que se confirma na presente investigação, que obteve o predomínio de $85 \%(51 / 60)$ do sexo masculino, e representando $47 \%(28 / 60)$ prevaleceu a faixa etária de 18 a 25 anos. Referente ao tempo de trabalho, constatou-se que $38 \%$ (23/60) dos entrevistados trabalham a menos de 1 ano na cerâmica. Relacionado ao turno de trabalho exercido pelos informantes, 93\% (56/60) informaram trabalhar durante os turnos manhã e tarde. A pesquisa demonstrou que $88 \%(53 / 60)$ dos trabalhadores, exercem suas atividades 8 horas por dia.

Na pesquisa pode-se observar que 15\% (9/60) dos trabalhadores possuem o hábito de fumar. Quanto ao setor laboral, 70\% (42/60) trabalham em serviços gerais, $22 \%$ (13/60) como forneiro. Quanto ao uso de EPI - máscara, 58\% (35/60) dos entrevistados relataram utilizar máscara no decorrer de suas atividades laborais.

$\mathrm{Na}$ ocorrência de sintomas, as variáveis mais relatadas pelos trabalhadores foram problemas respiratórios 37\% (22/60), visão embaralhada 32\% (19/60) e irritação nos olhos, garganta e narinas 30\% (18/60). 


\section{Níveis de carboxihemoglobina acima de 3,5\%}

Na pesquisa, 17\% (10/60) apresentaram níveis de $\mathrm{COHb}$ acima de $3,5 \%$, sendo todos do sexo masculino. Quanto à idade, prevaleceu a faixa etária entre 32 a 61 anos, correspondendo $40 \%$ (4/10) dos trabalhadores.

Referente ao tempo de trabalho, prevaleceram as variáveis entre 3 a 4 anos de trabalho na cerâmica, correspondendo a 30\% (3/10), e mais de 5 anos, com $30 \%$ (3/10). Quanto ao turno de trabalho, $90 \%(9 / 10)$ trabalham pela parte da manhã e tarde. Em relação à jornada diária trabalhada, 90\% (9/10) dos trabalhadores exercem suas atividades 8 horas por dia.

No presente estudo, como relatado na tabela 3, pôde-se observar que $60 \%$ (6/10) dos colaboradores, trabalham em serviços gerais e $40 \%(4 / 10)$ trabalham como forneiros. Com isso, observou-se que os trabalhadores de diferentes áreas foram afetados pelo $\mathrm{CO}$, com exceção do setor administrativo que tem suas instalações fora do ambiente de produção da cerâmica. Em um estudo realizado por Ferreira (2012), demonstrou que os setores de produção das cerâmicas estão sob influência do forno, equipamento que libera o $\mathrm{CO}$ através da queima de materiais que contenham carbono, e independente das funções que os trabalhadores exerçam, os mesmos estão expostos a este agente tóxico, com exceção dos que trabalham no setor administrativo que não sofrem influência direta do forno.

A análise da utilização do EPI - máscara demonstrou que $70 \%(7 / 10)$ dos entrevistados relataram fazer uso no decorrer de suas atividades laborais, sendo que esta é desprovida de filtros carregados de hopcalita. Desta forma, a máscara utilizada pelos trabalhadores nas cerâmicas pesquisadas, não são adequadas, pois em um estudo realizado por Varisco (2004), a eliminação do CO do ar inalado é feito a partir do uso de máscaras carregadas de hopcalita, que é uma mistura de óxidos metálicos $\left(\mathrm{Ag}_{2} \mathrm{O}, \mathrm{CO}_{2} \mathrm{O}_{3}, \mathrm{MnO}_{2}, \mathrm{CuO}\right)$ capaz de oxidar cataliticamente o $\mathrm{CO}$ à $\mathrm{CO}_{2}$.

Os sintomas característicos de exposição ao $\mathrm{CO}$, relatados pelos trabalhadores durante a pesquisa, estão apresentados na tabela 2.

Tabela 2 - Sintomas relatados pelos trabalhadores relacionados aos níveis de carboxihemoglobina

\begin{tabular}{|c|c|c|c|c|c|c|c|c|}
\hline \multirow[b]{3}{*}{ SINTOMAS } & \multicolumn{4}{|c|}{$\begin{array}{c}<3,5 \text { de COHb } \\
n=50\end{array}$} & \multicolumn{2}{|c|}{$\begin{array}{c}>3,5 \text { de } \\
\text { COHb } \\
n=10\end{array}$} & \multirow{2}{*}{\multicolumn{2}{|c|}{ Sim }} \\
\hline & \multicolumn{2}{|c|}{ Não } & \multicolumn{2}{|c|}{ Sim } & \multicolumn{2}{|c|}{ Não } & & \\
\hline & $n$ & $\%$ & $n$ & $\%$ & $n$ & $\%$ & $n$ & $\%$ \\
\hline Cefaléia & 38 & 76 & 12 & 24 & 9 & 90 & 1 & 10 \\
\hline Visão embaralhada & 34 & 68 & 16 & 32 & 7 & 70 & 3 & 30 \\
\hline Tontura & 39 & 78 & 11 & 22 & 10 & 100 & 0 & 0 \\
\hline Irritabilidade & 37 & 74 & 13 & 26 & 7 & 70 & 3 & 30 \\
\hline Diminuição da percepção visual & 39 & 78 & 11 & 22 & 10 & 100 & 0 & 0 \\
\hline
\end{tabular}

Saúde Meio Ambient. v. 4, n. 2, p. 90-102, jul./dez. 2015

ISSN 2316-347X 


\begin{tabular}{lllllllll}
\hline Cansaço & 36 & 72 & 14 & 28 & 8 & 80 & 2 & 20 \\
Taquicardia & 40 & 80 & 10 & 20 & 8 & 80 & 2 & 20 \\
Insônia & 46 & 92 & 4 & 8 & 8 & 80 & 2 & 20 \\
Hipertensão arterial & 46 & 92 & 4 & 8 & 8 & 80 & 2 & 20 \\
Precordialgia & 45 & 90 & 5 & 10 & 10 & 100 & 0 & 0 \\
Dislalia & 50 & 100 & 0 & 0 & 10 & 100 & 0 & 0 \\
Desmaio & 50 & 100 & 0 & 0 & 10 & 100 & 0 & 0 \\
Diminuição dos reflexos & 49 & 98 & 1 & 2 & 10 & 100 & 0 & 0 \\
Problemas respiratórios & 28 & 56 & 22 & 44 & 10 & 100 & 0 & 0 \\
Irritação nos olhos, garganta e narinas & 34 & 68 & 16 & 32 & 8 & 80 & 2 & 20 \\
Náusea & 47 & 94 & 3 & 6 & 9 & 90 & 1 & 10 \\
\hline
\end{tabular}

Gráfico 1 - Valores de carboxihemoglobina dos 10 trabalhadores que apresentaram níveis acima de $3,5 \%$

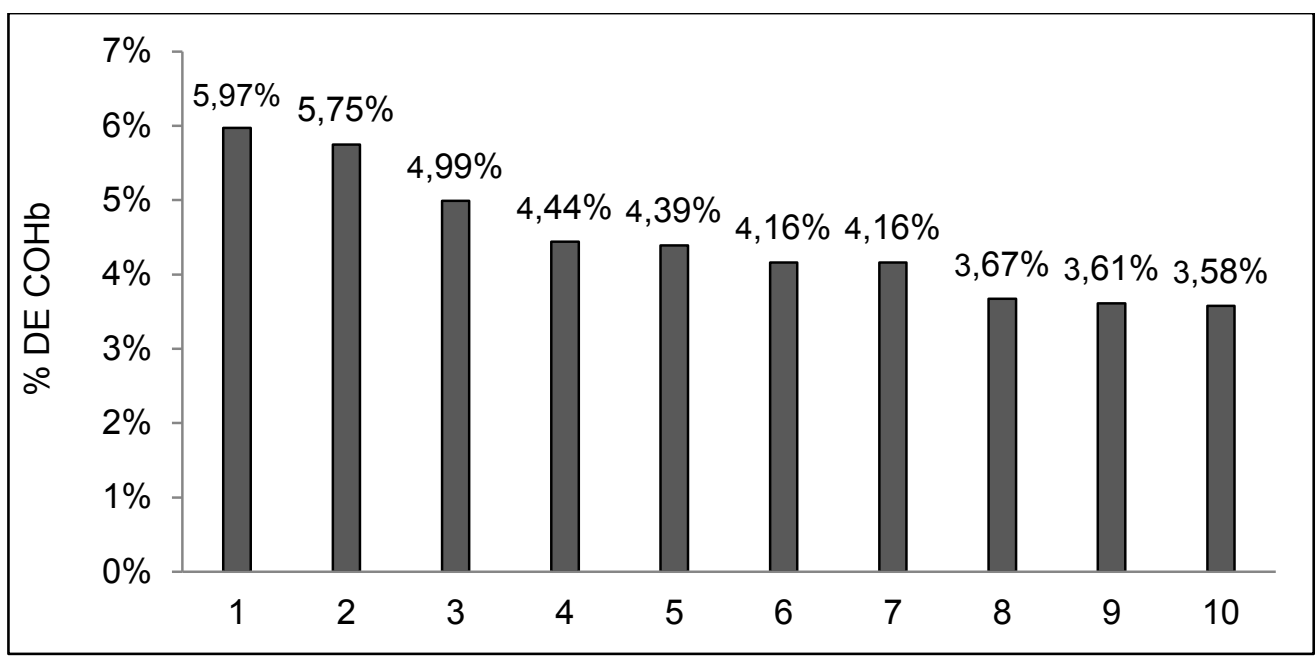

Referente à ocorrência de sintomas, conforme tabela 2, as variáveis mais relatadas pelos trabalhadores foram visão embaralhada $30 \%(3 / 10)$ e irritabilidade $30 \%$ (3/10). E em relação aos valores encontrados de COHb acima de 3,5\%, conforme gráfico 1 , observou-se que $30 \%(3 / 10)$ dos trabalhadores ficaram com níveis abaixo de $4 \%$, e $70 \%$ (7/10) dos trabalhadores obtiveram valores de $\mathrm{COHb}$ entre 4 a $6 \%$.

Estudo realizado por Peres (2005), afirma que os sintomas relacionados ao $\mathrm{CO}$, como diminuição da percepção visual e diminuição dos reflexos, começam a surgir quando os níveis de $\mathrm{COHb}$ chegam entre 4 a $6 \%$. No presente estudo, dos trabalhadores que apresentaram níveis entre 4 a $6 \%$, nenhum relatou apresentar os sintomas afirmados por Peres. Sibon et al.,(2007), em seu estudo, relata que os sintomas começam aparecer quando os níveis de $\mathrm{COHb}$ alcançam $10 \%$. $\mathrm{Na}$ presente pesquisa nenhum dos 10 trabalhadores que se encontraram com resultados de $\mathrm{COHb}$ acima de 3,5\% alcançaram níveis de $10 \%$, porém alguns trabalhadores relataram apresentar sintomas relacionados ao $\mathrm{CO}$. De acordo com o mesmo autor os sintomas de visão embaralhada e irritabilidade começam a surgir quando alcançam níveis de $\mathrm{COHb}$ entre 30 a 40\%, entretanto, no estudo observouse que nenhum dos colaboradores alcançaram níveis de 30 a $40 \%$, porém esses sintomas foram relatados por alguns trabalhadores. 
Os trabalhadores que apresentaram valores abaixo de $3,5 \%$ de $\mathrm{COHb}$, também relataram apresentar alguns possíveis sintomas relacionados a exposição ao $\mathrm{CO}$, subentendendo a partir desses dados, que essas possíveis sintomatologias poderiam estar relacionadas a outros fatores.

Gráfico 2 - Níveis de carboxihemoglobina relacionados ao hábito de fumar

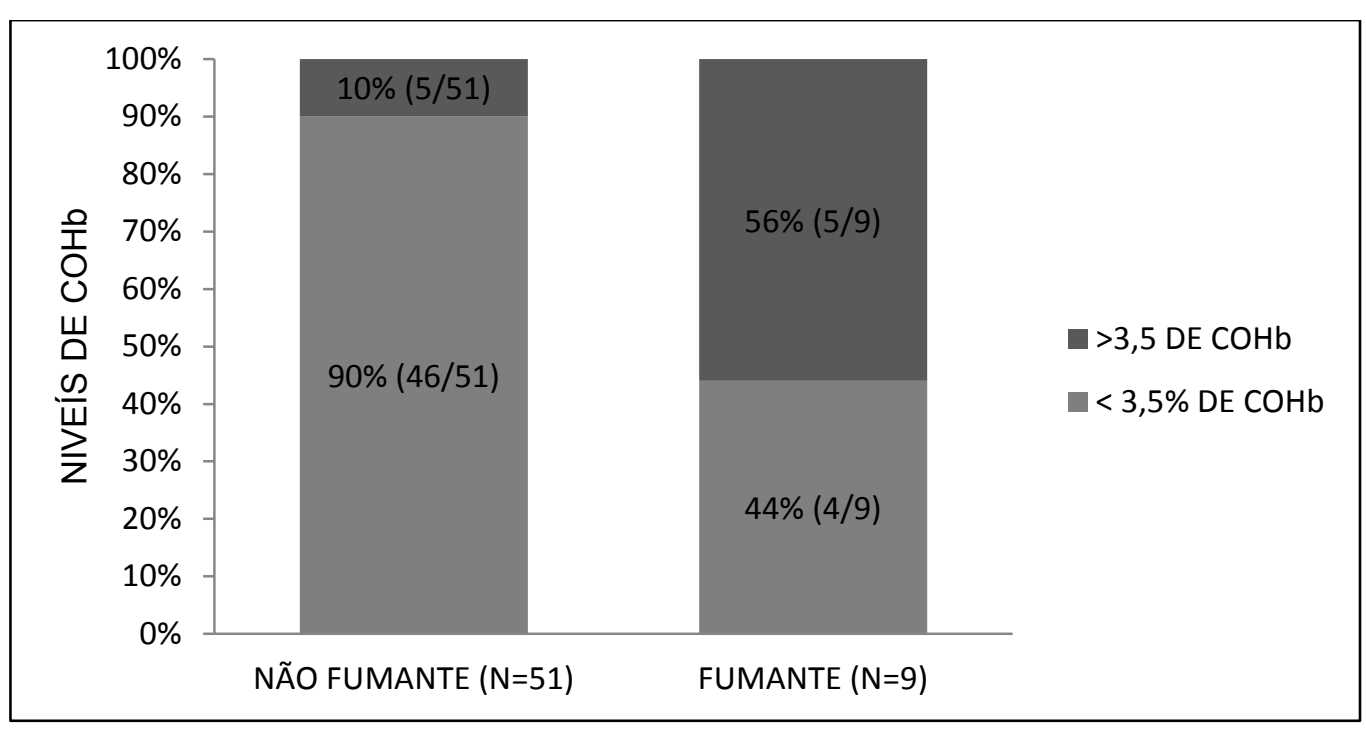

Em um estudo realizado por Silva (2012), demonstra que a fumaça do cigarro também é responsável pela emissão de CO, o que provavelmente coloca os fumantes em uma escala de risco maior, uma vez que, para eles a exposição tende a ser mais elevada, por já estarem em uma condição de exposição resultante do uso do cigarro. Tal afirmativa se apresenta no estudo, onde $56 \%$ (5/9), dos que relataram possuir o hábito de fumar, apresentaram níveis de $\mathrm{COHb}$ acima de 3,5\%, porém, foram encontrados valores acima de 3,5\% em trabalhadores que não possuem o hábito de fumar (Gráfico 2).

\section{CONSIDERAÇÕES FINAIS}

A partir do estudo realizado, foi possível observar que o CO é um produto tóxico de origem ambiental que traz sérios riscos a saúde. Sua liberação na indústria de cerâmica ocorre através do processo de queima de materiais orgânicos utilizados para a produção de telhas e tijolos, o que expõe seus trabalhadores a este agente tóxico.

A mensuração dos níveis de $\mathrm{COHb}$ nos trabalhadores mostrou que a maioria apresenta níveis dentro dos limites aceitáveis de acordo com o que preconiza a NR7. Portanto, considerando a possibilidade de exposição ao $\mathrm{CO}$, é importante manter 
um sistema periódico de monitoração, fazendo-se necessárias a elaboração e a implementação de um programa de acompanhamento e controle sistemático dos poluentes do ar e seus efeitos sobre a saúde humana, bem como, expor o estudo á vigilância sanitária do município de Santarém para que a mesma possa realizar fiscalizações constantes para que se tenha o cumprimento da legislação visando a qualidade de vida dos trabalhadores ceramistas.

Desta forma, novas pesquisas são necessárias para a ampliação do conhecimento dos níveis de $\mathrm{COHb}$ na população exposta ao $\mathrm{CO}$ em Santarém Pará, visto que este estudo é o primeiro relato sobre mensuração dos níveis de $\mathrm{COHb}$ na região.

\section{REFERÊNCIAS}

BEUTLER, E.; WEST, C. Simplified determination of carboxyhemoglobin. Clin. Chem., Whiston-Salem, v. 30, n. 6, p. 871-874, 1984. Disponível em: http://www.clinchem.org/content/30/6/871.full.pdf. Acesso em: 01 setembro 2012.

BOHM, Gyorgi Miklós. Poluição atmosférica: como os principais poluentes provocam doenças. Saúde Total. Disponível em: <www.saudetotal.com.br/artigos/ meioambiente/poluicao/spdoencpol.asp>. Acesso em: 10 fev. 2014.

CASTRO, H. A.; GOUVEIA, N.; CEJUDO, J. A. E. Questões metodológicas para a investigação dos efeitos da poluição do ar na saúde. Revista Brasileira de Epidemiologia, Rio de Janeiro, v.6, n.32, 2003. Disponível em: <http://www.scielo.br/pdf/rbepid/v6n2/07.pdf>. Acesso em: 05 out. 2012.

FERREIRA, Ruan Landolfo da Silva. Identificação e disposição final dos resíduossólidos gerados na fabricação de cerâmica vermelha no Vale do Assú/RN. 2012. 66f. Monografia (Graduação em Ciência e Tecnologia) Universidade Federal Rural do Semi - Árido. Campus Angicos. Disponível em: \&lt;http://www2.ufersa.edu.br/portal/view/uploads/setores/232/TCC\%20Ruan\%20Lan dolfo.pdf\&gt;. Acesso em: 07 mar. 2014.

GILMAN, Alfred Goodman. As bases farmacológicas da terapêutica. 10.ed. Rio de Janeiro: 2003.

GUYTON, Arthur C. Fisiologia humana. 6.ed. Rio de Janeiro: Guanabara Koogan, 2008.

GOMES, Marcos Hister Pereira. Manual de prevenção de acidente e doenças do trabalho nasolarias e cerâmicas vermelhas de Piracicaba e Região. Piracicaba SP, 2012. Disponível em: <http://www.cerest.piracicaba.sp.gov.br/site/images/ Manual-finalizado.pdf> Acesso em: 10 abr. 2013. 
LORENZI, Therezinha F. Manual de hematologia propedêutica e clínica. 4.ed. Rio de Janeiro: Guanabara Koogan, 2011.

MOREAU, Regina Lúcia de Moraes; SIQUEIRA, Maria Elisa Pereira Bastos. Toxicologia analítica. 1.ed. Rio de Janeiro: Guanabara, 2011.

OGA, Seizi; CAMARGO, Márcia Maria de Almeida; BATISTUZZO, Jose Antonio de Oliveira. Fundamentos de toxocologia. 2.ed. São Paulo: Atheneu, 2003.

OGA, Seizi; CAMARGO, Márcia Maria de Almeida; BATISTUZZO, Jose Antonio de Oliveira. Fundamentos de toxocologia. 3.ed. São Paulo: Atheneu, 2008.

PERES, Fábio de Farias. Os efeitos fisiológicos da poluição do ar no desempenho físico - o caso do monóxido de carbono (CO). Meio Ambiente e Saúde, Rio de Janeiro, v.1, n.1, 2005. Disponível em: <http://ergocenter.com.br/artigos/ artigos_2/meio_ambiente_e_saude.pdf>. Acesso em: 01 set. 2005.

PINA, Rosângela Zampieri; FURLAN, Maria Montserrat Diaz Pedrosa. Monóxido de carbono: uma nova molécula de sinalização celular, Maringá, v.3, n.1, 2007. Disponível: <http://www.mudi.uem.br/arqmudi/volume 11/numero 03/3-Pina.pdf> Acesso em: 28 abril 2013.

SALLES, Juliano Couto. Exposição ao monóxido de carbono: as alterações cardiovasculares no organismo humano. 2013. 31f. Monografia (Graduação em Medicina) - Faculdade de Medicina da Bahia, Universidade Federal da Bahia, Salvador. Disponível em: <http://repositorio.ufba.br/ri/handle/ri/13364>. Acesso em: 05 mar. 2014.

SIBON, A. Olano; et al. Intoxicação por monóxido de carbono. Jornal de Medicina Legal, Sevilla, n. 47. 2007. Disponível em:<http://scielo.isciii.es/scielo.php?pid= S113576062007000100007\&script=sci_arttext\&tlng=pt> Acesso em 12 out.2007.

SILVA, Luiz Almeida da. Exposição ambiental ao monóxido de carbono e acidentes de trabalho entre mototaxistas: uma contribuição da enfermagem do trabalho, 2012, 203f. Tese (Pós-Graduação em Enfermagem Fundamental) - Escola de Enfermagem, Universidade de São Paulo, Ribeirão Preto.Disponível em: $<$ http://www.teses.usp.br/teses/disponiveis/22/22132/tde-17042013$143634 / \& \mathrm{~cd}=3 \&$ ved=0CDEQFjAC\&usg=AFQjCNHbplGIHaLGs9UvLkRfZFFFPUM8Q\&sig2=xkG6jkpMG732ae2CyQ6noQ>. Acesso em: 11 abr. 2014.

SOUSA, Ayda Tieko Ajisaka. Perfil da função respiratória de trabalhadores na indústria de cerâmica de São Miguel do Guamá, Belém, 2009. Disponível em: <http://www.unama.br/novoportal/ensino/graduação/cursos/fisioterapia/attachments/ article/131/perfil_função_respiratoria_trabalhadores_ceramica_sao_miguel_guama.p df> Acesso em: $\mathbf{2} 5$ abr. 2013. 
VARISCO, Maria Caroline. Avaliação da exposição ocupacional ao monóxido de carbono em trabalhadores de postos de combustíveis. 2004. 54f. Monografia (Graduação em Ciências farmacêuticas) - Centro Universitário de Feevale, Instituto de Ciências da Saúde, Novo Hamburgo. Disponível em:

$<$ http://ged.feevale.br/bibvirtual/Monografia/MonografiaMariaVarisco.pdf. Acesso em: 08 outubro 2012.

Artigo recebido em: 14/04/2015

Artigo aprovado em: 25/11/2015 